\title{
IMPROVEMENT OF THE ELECTRICAL PROPERTIES OF SYNTHETIC LIQUID DIELECTRIC FOR PULSE CAPACITORS
}

\author{
Maya Yadigar Abdullayeva \\ Department of Chemical Technology ${ }^{l}$ \\ mayaabdullayeva@hotmail.com \\ Ibrahim Abulfas Habibov \\ Department of Engineering Drawing ${ }^{l}$ \\ h.ibo@mail.ru \\ Azerbaijan State University of Oil and Industry \\ 20 Azadlig ave., Baku, Azerbaijan, AZ 1010
}

\begin{abstract}
An increase in electricity generation is possible not only through the construction of power plants, but also through the creation of fundamentally new energy sources. One of the problems of modern electrical engineering is to ensure the reliability of the operation of capacitors at electric field strengths exceeding I50-200MV. In the domestic and foreign capacitor industry, for heavy pulse modes, castor oil (CO) is used as a liquid impregnation. The development of a method for producing a substitute for natural castor oil, which is a universal impregnating liquid in capacitors, is an urgent problem in the electrical industry. Thus, we have developed a method for the production of acetoxymethyl-w.hexyl-o-xylene, the electrophysical properties of which make it possible to use it as an environmentally friendly and promising substitute for natural castor oil. However, there was a problem of stabilization, since compounds of the ester type have (as impregnating liquids in capacitors) a disadvantage, which consists in their sensitivity to light hydrolysis and atmospheric effects. Ester exhibits high stability with respect to air up to $200{ }^{\circ} \mathrm{C}$, therefore, inhibition by the addition of antioxidants is required is not new.

In this work developed the basic electrophysical properties of the ester-acetoxymethyl-secondary hexyl-o-xyleole; methods for its purification and stabilization. There were chosen the adsorption method of thermo-oxidative stabilization to clean dielectric fluid from conductive impurities.

As a result of the electrophysical characteristics of the acetoxymethyl-secondary hexyl-o-xyleole ester, as well as the method of its purification using alumina, and hydrogenation on a catalyst representing $0.2 \%$ palladium on alumina and stabilization using additives NG-2246. As a result of the research, it was possible to obtain an ester with improved electrophysical parameters.
\end{abstract}

Keywords: hydrogenation, dehydration, dielectric liquid, stabilization, aluminum oxide, adsorbent, castor oil.

DOI: $10.21303 / 2461-4262.2020 .001519$

\section{Introduction}

Esters are used in power capacitors used in electrical power engineering, industrial electrical engineering, and power electronics. In addition, esters are promising substitutes for chlorobiphenyls, dielectric fluids, which are prohibited in many countries due to their toxicity and biodegradability, which pose a serious environmental hazard [1-5].

Known synthetic esters, as well as castor oil, along with a wide range of valuable physical and chemical properties, have insufficient hydrolytic stability, which sharply reduces the service life of power capacitors. Therefore, the known esters used as dielectric liquids require stabilization with epoxy additives that block acid groups and hinder their hydrolysis.

Despite the fact that insufficient hydrolytic stability of esters is a generally recognized factor [6], as follows from the contents of Table $1[6,7]$, the dielectric liquid acetoxymethyl-secondary hexyl-o-xyleole, obtained in strict accordance with the methodology of its synthesis, is thermostable.

However, in the process of impregnation, a situation is not excluded when small deviations from the given technological regime are possible, leading to the appearance of undesirable impurities in the final regime. If to add here possible violations of the rules of storage in a warehouse or transportation, then it is really necessary to take into account the appearance of such impurities, arising, for example, in the processes of hydrolysis or photolysis of a dielectric liquid. In this regard, 
in order to obtain guaranteed stable results, it became necessary to develop methods for drying a dielectric liquid from conductive impurities.

Table 1

Physicochemical and electrical characteristics of the dielectric liquid acetoxymethyl-secondary hexyl-o-xyleole

\begin{tabular}{lcc}
\hline Description of characteristics & Acetoxymethyl-secondary hexyl-o-xylene* & Indian medical castor oil \\
\hline Density at $20^{\circ} \mathrm{C}, \mathrm{kg} / \mathrm{m}^{3}$ & 965 & 958 \\
Kinematic viscosity, $\mathrm{m}^{2} / \mathrm{c}$ & & About 1,000 \\
at $20^{\circ} \mathrm{C}$ & 44.68 & 56 \\
at $70{ }^{\circ} \mathrm{C}$ & 5.92 & 1.4780 \\
Degree of refraction & 1.4975 & 275 \\
Flash point, ${ }^{\circ} \mathrm{C}$ & 162 & 0.0014 \\
The loss tangent of a dielectric & & 0.66 \\
at $20^{\circ} \mathrm{C}$ & 0.002 & 4.52 \\
at $90^{\circ} \mathrm{C}$ & 0.02 & 3.90 \\
The dielectric constant & &
\end{tabular}

Note: * - without additional cleaning, drying and stabilization.

\section{Materials and research methods}

In order to obtain high and stable electrophysical parameters of the dielectric liquid acetoxymethyl-secondary hexyl-o-xyleole, the main of which are high dielectric constant and resistance to electrical breakdown, various options for drying and purification were studied [8].

Electrical indicators characterizing the presence of ionic and conductive impurities in the liquid were measured in accordance with GOST 6581-75 at temperatures of 20 and $90{ }^{\circ} \mathrm{C}$. These indicators include: the tangent of the angle of dielectric loss $(\operatorname{tg} \delta)$, specific volume resistance $\left(\rho_{v}\right)$, electrical permeability $(\varepsilon)$.

Table 2 shows the method for determining the physicochemical and electrophysical properties of acetoxymethyl-secondary hexyl-o-xyleole.

Table 2

Method for determining the physicochemical and electrophysical properties of acetoxymethyl-secondary hexyl-o-xyleole

\begin{tabular}{lccc}
\hline \multicolumn{1}{c}{ Index } & Symbol & Measurement & Determination method according to GOST \\
\hline Pour point & $T_{\text {point }}$ & $\mathrm{K}$ & $20287-78$ \\
Boiling temperature & $T_{\text {boil }}$ & $\mathrm{K}$ & $18995-6-73$ \\
Flash point & $T_{f l}$ & $\mathrm{~K}$ & $4333-48$ \\
Refractive index & $n_{D}$ & dimensionless & Refractometer IRF-20 \\
Density & $\rho_{4}^{20}$ & $\mathrm{~kg} / \mathrm{m}^{3}$ & $1300-74$ \\
Kinematic viscosity & $v$ & $\mathrm{~m}^{2} / \mathrm{s}-1 \cdot 10^{6}$ & $33-82$ \\
Heat capacity & $C p$ & $\mathrm{~J} /(\mathrm{kg} \cdot \mathrm{K})$ & $\begin{array}{c}\text { Method of an adiabatic flow-through calorimeter in a closed cir- } \\
\text { culation circuit with a weight measurement of the flow rate }\end{array}$ \\
The loss tangent of a dielectric & $\operatorname{tg} \delta$ & $\%$ & $6581-75$ \\
Specific volume resistance & $\rho$ & $\mathrm{Om} \cdot \mathrm{m}$ & $6581-75$
\end{tabular}


The electrophysical and physicochemical properties of the synthesized acetoxymethylsecondary hexyl-o-xyleole, were determined by standard methods after vacuum distillation and fine purification from conductive impurities by the adsorption method on aluminum oxide [9].

The thermal stability of dielectric liquid was determined by the increase in the value $(\operatorname{tg} \delta)$ of the liquid after holding it at $90{ }^{\circ} \mathrm{C}$ for 48 hours. If the increase (tg $\delta$ ) with respect to the initial one was $\%$, then the liquid was considered thermostable.

\section{Results}

Purification of the synthesized ether from conductive impurities was carried out by the adsorption method in a closed system. The installation for drying the dielectric consisted of a one-liter flask with a sampling branch and a glass stopper connected to a three-way valve. A liquid was placed in the flask and pre-prepared alumina was added thereto in an amount of $10 \%$ by weight of the liquid to be purified. To avoid undesirable contacts of the dielectric with atmospheric oxygen, the flask was filled with nitrogen, evacuated, and then nitrogen was supplied again through a three-way valve. At the same time, the liquid was uniformly mixed with aluminum oxide. Then the closed flask was disconnected from the vacuum pump and kept at a temperature of $90^{\circ} \mathrm{C}$ for several hours.

A reactor equipped with a porous glass filter, nichrome wire winding and a reflux condenser was used for hydrogenation. The reactor consisted of a Pyrex glass tube with a diameter of $30 \mathrm{~cm}$ and a length of $50 \mathrm{~cm}$, a pocket for a thermocouple and two taps for supplying hydrogen from the bottom and for loading and unloading a dielectric liquid and catalyst from above. A reflux condenser was connected to the upper outlet during hydrogenation.

The catalyst used was $0.2 \%$ palladium on alumina in an amount of $5 \%$ of the dielectric volume. Hydrogen was fed from the generator at a rate of $40 \mathrm{ml} / \mathrm{min}$ through a desiccant filled with aluminum oxide, the reaction temperature was $120^{\circ} \mathrm{C}$. After hydrogenation, the reaction mixture was washed to a neutral medium, dried over sodium sulfate, distilled, and purified over $\gamma-\mathrm{Al}_{2} \mathrm{O}_{3}$.

In order to obtain high and stable electrophysical characteristics of the dielectric liquid secondary hexyl-o-xyleole, the main of which are high dielectric constant and resistance to electrical breakdown, various options for drying and chemical purification were investigated [10, 11]. $\gamma$-aluminum oxide (fraction 1-1.5 mm) was used as an adsorbent. The drying results are shown below (Table 3).

As can be seen from the test results, short-term drying up to 4 hours improves the electrophysical characteristics of the dielectric liquid. Prolonged contact of it with the adsorbent, obviously, causes the destruction of its structure. The adsorbent is eroded and converted into a pulverized state; which ultimately leads to the ingress of small particles, which are difficult to remove during filtration, into the dielectric liquid, which sharply reduces its electrophysical characteristics.

Thus, it was assumed that insufficient thermal stability of the dielectric liquid as a result of partial hydrolysis of traces of chloromethyl- secondary hexyl-o-xyleole, in the composition of the dielectric liquid leads to the appearance of the corresponding hydroxy-methyl derivative is shown in Fig. 1.

Table 3

Influence of the drying time on the electrophysical parameters of acetoxymethyl-secondary hexyl-o-xyleole

\begin{tabular}{|c|c|c|c|c|c|c|}
\hline \multirow{2}{*}{ Electrophysical indicators } & \multirow{2}{*}{ Before drying } & \multicolumn{5}{|c|}{ Drying time, hour } \\
\hline & & 2 & 4 & 6 & 8 & 10 \\
\hline \multicolumn{7}{|l|}{ The loss tangent of a dielectric } \\
\hline by $20^{\circ} \mathrm{C}$ & 0.047 & 0.003 & 0.003 & 0.005 & 0.042 & 0.071 \\
\hline by $90^{\circ} \mathrm{C}$ & 0.042 & 0.003 & 0.03 & 0.06 & 0.054 & 0.073 \\
\hline by $90^{\circ} \mathrm{C}$ after 48 minutes & 0.049 & 0.003 & 0.03 & 0.07 & 0.08 & 0.079 \\
\hline \multicolumn{7}{|l|}{ The dielectric constant } \\
\hline by $20^{\circ} \mathrm{C}$ & 5.3 & 5.2 & 5.1 & 5.3 & 5.5 & 5.7 \\
\hline by $90^{\circ} \mathrm{C}$ & 4.7 & 4.5 & 4.3 & 4.5 & 4.7 & 4.8 \\
\hline by $90^{\circ} \mathrm{C}$ after 48 minutes ${ }^{\circ} \mathrm{C}$ & 4.7 & 4.5 & 4.2 & 4.4 & 4.6 & 4.7 \\
\hline
\end{tabular}




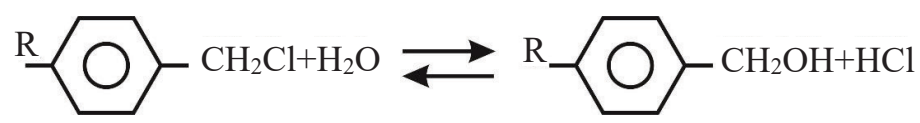

Fig. 1. Hydrolysis scheme chloromethyl- secondary hexyl-o-xyleole

The dielectric liquid was purified by hydrogenation. Installation for purification of a dielectric liquid by hydrogenation is shown in Fig. 2.

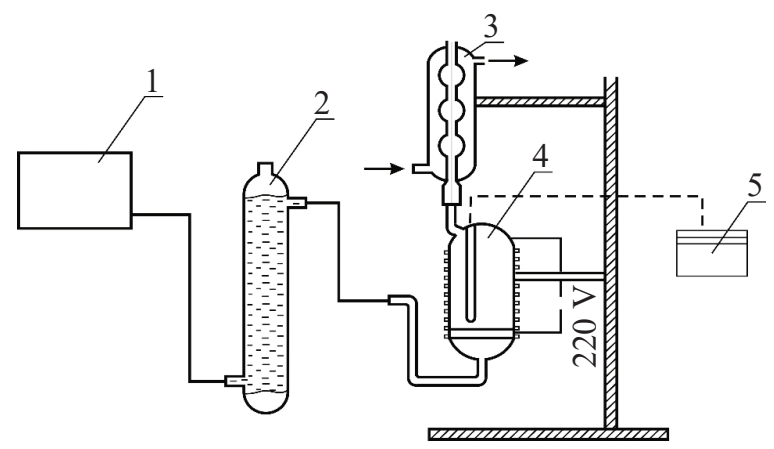

Fig. 2. Dielectric fluid hydrogenation plant:

1 - hydrogen generator, 2 - desiccant, 3 - refrigerator, 4 - reactor, 5 - laboratory transformer

For hydrogenation, a reactor (4) equipped with a porous glass filter, nichrome wire winding, and a reflux condenser (3) was used. The reactor consisted of a pyrex glass tube with a diameter of $30 \mathrm{~cm}$ and a length of $50 \mathrm{~cm}$, a pocket for a thermocouple, and two taps for supplying hydrogen from the bottom and for loading and unloading the dielectric liquid and catalyst from above. A reflux condenser was connected to the upper outlet during hydrogenation.

\section{Discussion of experimental results}

Experiments show that this treatment improves the thermal stability of the dielectric fluid. The catalyst used was $0.2 \%$ palladium on alumina in an amount of $5 \%$ by volume of the dielectric. Hydrogen from the generator (1) was fed at a rate of $40 \mathrm{ml} / \mathrm{min}$ through a desiccant (2) filled with aluminum oxide, the reaction temperature was $120^{\circ} \mathrm{C}$. After hydrogenation, the reaction mixture was washed until neutral, dried over sodium sulfate, distilled and subjected to $\gamma-\mathrm{Al}_{2} \mathrm{O}_{3}$ purification. The electro physical parameters of the liquid after hydrogenation are presented in Table 4.

As can be seen from Table 4, almost 4 hours after the start of hydrogenation, the thermal stability significantly increases, the value of the dielectric loss tangent decreases, the dielectric constant and specific volume resistance change slightly.

As can be seen from Table 4, almost 4 hours after the start of hydrogenation, the thermal stability significantly increases, the value of the dielectric loss tangent decreases, the dielectric constant and specific volume resistance change slightly.

Thermo-oxidative stability of an insulating liquid characterizes its resistance to temperature and oxidation when in contact with atmospheric oxygen. An indicator of thermo-oxidative stability is the magnitude of the change in the tangent of the dielectric loss angle at a temperature of $80-100{ }^{\circ} \mathrm{C}$. To improve the performance of dielectric fluids, it is usually necessary to stabilize them with additives.

For this purpose, the effect of an industrial antioxidant additive on the properties of acetoxymethyl- secondary hexyl-o-xyleole, was studied. As a criterion of stability, the change in the tangent of the dielectric loss angle was taken when heated to $90{ }^{\circ} \mathrm{C}$ for 48 hours. An industrial phenolic antioxidant 2,2-methylene-bis-6-tert-butyl-4-methyl-phenol (NG-2246) [12] was used as an additive.

Table 5 shows the results of stabilization of acetoxymethyl-secondary hexyl-o-xyleole, with additive - NG-2246. The change in the dielectric loss tangent upon heating to $90{ }^{\circ} \mathrm{C}$ for 48 hours was taken as a stability criterion. 
Table 4

Influence of the duration of hydrogenation of the dielectric liquid acetoxymethyl-secondary

hexyl-o-xyleole, on its electrophysical parameters

\begin{tabular}{|c|c|c|c|c|}
\hline \multirow{2}{*}{ Electrophysical indicators } & \multirow{2}{*}{ Initial data } & \multicolumn{3}{|c|}{ After hydrogenation, hour } \\
\hline & & 2 & 4 & 6 \\
\hline \multicolumn{5}{|l|}{ Specific volume resistance, $\mathrm{Ohm} \cdot \mathrm{m}$} \\
\hline by $20^{\circ} \mathrm{C}$ & $1.57 \cdot 10^{12}$ & $1.55 \cdot 10^{12}$ & $1.72 \cdot 10^{12}$ & $1.32 \cdot 10^{12}$ \\
\hline by $90^{\circ} \mathrm{C}$ & $1.27 \cdot 10^{10}$ & $1.22 \cdot 10^{10}$ & $8.5 \cdot 10^{10}$ & $1.44 \cdot 10^{10}$ \\
\hline by $90{ }^{\circ} \mathrm{C}$ after 48 minutes & $1.3 \cdot 10^{10}$ & $1.27 \cdot 10^{10}$ & $8.9 \cdot 10^{10}$ & $1.51 \cdot 10^{10}$ \\
\hline \multicolumn{5}{|l|}{ The dielectric constant } \\
\hline by $20^{\circ} \mathrm{C}$ & 5.4 & 5.3 & 5.2 & 5.1 \\
\hline by $90^{\circ} \mathrm{C}$ & 4.8 & 4.5 & 4.3 & 4.2 \\
\hline by $90^{\circ} \mathrm{C}$ after 48 minutes & 4.8 & 4.4 & 4.2 & 4.2 \\
\hline \multicolumn{5}{|l|}{ The loss tangent of a dielectric } \\
\hline by $20^{\circ} \mathrm{C}$ & 0.13 & 0.008 & 0.004 & 0.002 \\
\hline by $90^{\circ} \mathrm{C}$ & 0.20 & 0.06 & 0.02 & 0.02 \\
\hline by $90^{\circ} \mathrm{C}$ after 48 minutes & 0.27 & 0.08 & 0.04 & 0.03 \\
\hline
\end{tabular}

Table 5

Effect of additive NG-2246 on thermal oxidative stability of acetoxymethyl- secondary hexyl-o-xyleole

\begin{tabular}{|c|c|c|c|}
\hline \multirow{2}{*}{ Electrophysical indicators } & \multicolumn{3}{|c|}{ Amount of NG-2246, wt. \% } \\
\hline & 0.005 & 0.01 & 0.05 \\
\hline \multicolumn{4}{|l|}{ Specific volume resistance, $\mathrm{Om} \cdot \mathrm{m}$} \\
\hline by $20^{\circ} \mathrm{C}$ & $1.72 \cdot 10^{12}$ & $1.81 \cdot 10^{12}$ & $1.78 \cdot 10^{12}$ \\
\hline by $90^{\circ} \mathrm{C}$ & $5.28 \cdot 10^{11}$ & $6.11 \cdot 10^{11}$ & $5.35 \cdot 10^{11}$ \\
\hline by $90{ }^{\circ} \mathrm{C}$ after 48 minutes & $5.21 \cdot 10^{10}$ & $5.35 \cdot 10^{10}$ & $5.20 \cdot 10^{10}$ \\
\hline \multicolumn{4}{|l|}{ The dielectric constant } \\
\hline by $20^{\circ} \mathrm{C}$ & 5.0 & 5.0 & 5.0 \\
\hline by $90^{\circ} \mathrm{C}$ & 4.3 & 4.2 & 4.2 \\
\hline by $90^{\circ} \mathrm{C}$ after 48 minutes & 4.2 & 4.1 & 4.1 \\
\hline \multicolumn{4}{|l|}{ The loss tangent of a dielectric } \\
\hline by $20^{\circ} \mathrm{C}$ & 0.003 & 0.002 & 0.006 \\
\hline by $90^{\circ} \mathrm{C}$ & 0.003 & 0.002 & 0.008 \\
\hline by $90^{\circ} \mathrm{C}$ after 48 minutes & 0.004 & 0.003 & 0.010 \\
\hline
\end{tabular}

As can be seen from the results, the additive NG-2246 in an amount (0.01 wt \%) increases the thermal-oxidative stability of the dielectric fluid. As a result of biomedical and toxicological studies of acetoxymethyl- secondary hexyl-o-xyleole, it has been established that this liquid belongs to class IV (i.e., it is low-toxic).

\section{Conclusion}

In this work, as a result of the conducted studies of the electrophysical parameters of the ester of acetoxymethyl-secondary hexyl-o-xyleole, a method for its purification using aluminum oxide was chosen, and hydrogenation was carried out on a catalyst representing $0.2 \%$ palladium on alumina and stabilization using additive NG-2246. 
An ester of acetoxymethyl-secondary hexyl-o-xyleole with improved electrophysical indicators has been obtained, namely, thermal stability increases, the value of the dielectric loss tangent decreases, and the dielectric constant and specific volume resistance change slightly.

\section{References}

[1] Perrier, C., Beroual, A. (2009). Experimental investigations on insulating liquids for power transformers: Mineral, ester, and silicone oils. IEEE Electrical Insulation Magazine, 25 (6), 6-13. doi: https://doi.org/10.1109/mei.2009.5313705

[2] Ruder, A. M., Hein, M. J., Hopf, N. B., Waters, M. A. (2014). Mortality among 24,865 workers exposed to polychlorinated biphenyls (PCBs) in three electrical capacitor manufacturing plants: A ten-year update. International Journal of Hygiene and Environmental Health, 217 (2-3), 176-187. doi: https://doi.org/10.1016/j.ijheh.2013.04.006

[3] Kodavanti, P. R. S. (2017). Polychlorinated Biphenyls (PCBs). Reference Module in Neuroscience and Biobehavioral Psychology. doi: https://doi.org/10.1016/b978-0-12-809324-5.03955-9

[4] Moore, S. P. (2006). Some Considerations for New and Retrofil Applications of Natural Ester Dielectric Fluids in Medium and Large Power Transformers. 2005/2006 PES TD. doi: https://doi.org/10.1109/tdc.2006.1668447

[5] Azis, N. (2012). Ageing Assessment of Insulation Paper with Consideration of In-Service Ageing and Natural Ester Application. Manchester, UK: The University of Manchester, 213.

[6] Sarel, S., Tsai, L., Newman, M. S. (1956). Rates of Alkaline Hydrolysis of a Series of Primary and Secondary Alkyl Acetates. Journal of the American Chemical Society, 78 (20), 5420-5423. doi: https://doi.org/10.1021/ja01601a069

[7] Abdullayeva, M. Y., Gasanov, A. A. (2018) About technically useful properties of esters on the base of alkylaromatic hydrocarbons. Bulletin of Science and Practice, 4 (11), 12-22. Available at: https://cyberleninka.ru/article/n/about-technically-useful-properties-of-esters-on-the-base-of-alkylaromatic-hydrocarbons

[8] Wang, Z., Darwin, A., Martin, R. (2007). New insulation fluids: use of environmentally friendly fluids in power transformers. Proc. of CIGRE colloquium, Brugge.

[9] Anikeeva, M. A., Korobeynikov, S. M. (2016). Study of stability against oxidation of rapeseed oil. Journal of Engineering Thermophysics, 25 (2), 236-238. doi: https://doi.org/10.1134/s1810232816020089

[10] Peralta-Robledo, R. E., Santolalla-Vargas, C. E., Sanchez-Minero, F., Santes, V., Flores-Valle, S. O., Elizalde Solis, O. (2020). Effect of the formulation of $\mathrm{Pd} / \gamma-\mathrm{A} 12 \mathrm{O} 3+\mathrm{Pd} / \mathrm{H}-\mathrm{ZSM}-5$ catalysts prepared by mechanical mixing for the thermal and catalytic hydrotreating of castor oil. Catalysis Today, 346, 81-86. doi: https://doi.org/10.1016/j.cattod.2019.02.063

[11] Lisitsyn, A. S., Parmon, V. N., Duplyakin, V. K., Likholobov, V. A. (2006). Modern Problems and Prospects for the Development of Investigations in the Field of Preparation of Deposited Palladium Catalysts. Ross. Khim. Zh., 50 (4), 140-153.

[12] Edenbaum, J., Reinhold, V. N. (Eds.) (1992). Plastics additives and modifiers handbook. New York, 1113.

Received date 03.10.2020

Accepted date 13.11.2020

Published date 30.11.2020
(C) The Author(s) 2020

This is an open access article under the CC BY license (http://creativecommons.org/licenses/by/4.0). 